\title{
Recent Advances in Modeling Hugoniots with Cheetah
}

K. R. Glaesemann, L. E. Fried

July 28,2005

14th APS Topical Conference on Shock Compression of Condensed Matter Baltimore, MD, United States July 31, 2005 through August 5, 2005 
This document was prepared as an account of work sponsored by an agency of the United States Government. Neither the United States Government nor the University of California nor any of their employees, makes any warranty, express or implied, or assumes any legal liability or responsibility for the accuracy, completeness, or usefulness of any information, apparatus, product, or process disclosed, or represents that its use would not infringe privately owned rights. Reference herein to any specific commercial product, process, or service by trade name, trademark, manufacturer, or otherwise, does not necessarily constitute or imply its endorsement, recommendation, or favoring by the United States Government or the University of California. The views and opinions of authors expressed herein do not necessarily state or reflect those of the United States Government or the University of California, and shall not be used for advertising or product endorsement purposes. 


\title{
RECENT ADVANCES IN MODELING HUGONIOTS WITH CHEETAH
}

\author{
K. R. Glaesemann ${ }^{1}$ and L. E. Fried ${ }^{1}$ \\ ${ }^{I}$ Chemistry and Materials Science Directorate, Lawrence Livermore National Laboratory, L-282, P.O. Box 808, \\ Livermore CA 94551
}

\begin{abstract}
We describe improvements to the Cheetah thermochemical-kinetics code's equilibrium solver to enable it to find a wider range of thermodynamic states. Cheetah supports a wide range of elements, condensed detonation products, and gas phase reactions. Therefore, Cheetah can be applied to a wide range of shock problems involving both energetic and non-energetic materials. An improve equation of state is also introduced. New experimental validations of Cheetah's equation of state methodology have been performed, including both reacted and unreacted Hugoniots
\end{abstract}

Keywords: Cheetah, thermochemistry, equation of state, Hugoniot.

PACS: 82.20.-w, 82.33.Vx, 47.40.-x, 95.30.Tg.

\section{INTRODUCTION}

Cheetah [1] is a thermochemical-kinetics code based upon solving chemical equilibrium or kinetic equations. Cheetah is used for the development, characterization, and assessment of explosives, pyrotechnics, gun and rocket propellants, materials, and planet interiors [2]. The gas equation of state (EOS) is an exponential-6 model of interacting fluids [3]. The condensed EOS is an enhanced Murnaghan EOS [4]. Any thermodynamic potential or thermodynamic second derivative can be conveniently calculated. The solution state can be constrained by physical properties: volume $\mathrm{V}$ (equivalently: density $\rho$ ), energy $\mathrm{E}$, entropy $\mathrm{S}$, temperature T, Helmholtz free energy A, pressure P, enthalpy H, Gibbs free energy G, the Hugoniot, or the Rayleigh line.

To maintain code quality, an extensive automatic test suite has been developed to verify code functionality. The suite consists of over 300 Cheetah runs, with many including multiple calculations within them. Results are automatically compared to archived results. Scripts regularly scan the source code and verify that all commands are in the test suite.

The aforementioned test suite verifies that Cheetah gives the expected result, but does not verify that the expected result is physically accurate. Another test suite, called "fits", is used for this purpose. The fits system compares Cheetah to more than 3000 unique experimental data points. More specifically, the suite contains as of this report: 147 Hugoniots fits (both reacted and unreacted), 7 shock temperature fits, 9 re-shock Hugoniot fits, 12 isothermal compression fits, 13 sound speed fits, 126 heat capacity fits, and 24 phase diagram fits. The test suites (in particular the Hugoniot data), along with user needs, has driven significant improvements to both the methodologies in the code and the EOS library.

\section{IMPROVEMENTS TO THE EOS}

Cheetah 4.0 includes version 2 of the exponential 6 EOS. Version 2 completed the stoichiometric set for most elements used (solid, liquid and gas states). To improve accuracy, new chemical species, such as $\mathrm{FeS}_{2}$, have been added. For 
species where multiple solid states exist, such as $\mathrm{Fe}_{3} \mathrm{O}_{4}$ and phosphorous, the EOS now includes multiple solid phases. Some materials had liquid phases added, such as $\mathrm{Ti}_{2} \mathrm{O}_{3}$. In addition to enlarging the EOS library, the fits test suite allowed careful optimization of the EOS. The details of these improvements will be the subject of a later work, while this work will focus more on computational improvements to the Cheetah code.

\section{IMPROVED SOLUTIONS OF STATES}

The equilibrium solver in Cheetah 3.0 could not easily handle all possible states. Unfortunately, the limitations were not obvious, because they were dependent upon the chemical system. In this work, we describe advances in Cheetah 4.0 which allow it to solve a wider range of problems than 3.0.

The interface to the existing equilibrium solver has been significantly enhanced. Cheetah 4.0 makes a substantially better initial guess at the target thermodynamic state. The first step in this process is to set the $\mathrm{V}, \mathrm{T}$, and/or $\mathrm{P}$ to the requested values. Since at most two of these can be specified, unspecified $\mathrm{V}, \mathrm{T}$ and $\mathrm{P}$ are set to the last calculated state's value (for the first state with this composition, they are set to reasonable generic values). If two are specified, then the third is found iterately. If only $\mathrm{V}$ is specified then $\mathrm{P}$ is found. For all other states, V is found which is consistent with $\mathrm{P}$ and $\mathrm{T}$.

Cheetah 4.0 has also significantly improved algorithms for "sneaking up" on hard to solve thermodynamic states. This provides Cheetah with better recovery from initial failure. Cheetah tries successively smaller steps from the current state to the target state.

These two subtle, but important, enhancements have resulted in many states converging that would not easily converge in Cheetah 3.0.

Cheetah requires a value of gaseous $\mathrm{P}$, thus low gas states were problematic in Cheetah 3.0. This resulted in the inability to run thermites. Cheetah 4.0 adds infinitesimal quantities of generic gas, thus guaranteeing that $\mathrm{P}$ is defined. The amount is set small enough to avoid affecting properties. In Cheetah 3.0, the user could add Argon to achieve a similar effect, but it did change the results.

A new solver that minimizes free energy has been implemented. Although only the Donlp2
SQP solver [5] is implemented, a generic interface allows other solvers to easily be added. We have modified Donlp2 to verify solution validity. Only state types for with a corresponding thermodynamic potential exists can be solved (V and T; $\mathrm{P}$ and $\mathrm{T} ; \mathrm{V}$ and $\mathrm{E} ; \mathrm{S}$ and $\mathrm{V} ; \mathrm{S}$ and $\mathrm{P} ; \mathrm{P}$ and $\mathrm{H})$. Although the Hugoniot is not directly solvable, the Donlp2 method is a user selectable option for solving the initial state point, which can be troublesome. For example, the following calculation does not solve directly without using Donlp2 [6]:

comp,ap,20,rdx,25,al,33,nto,10,htpb,10.5,n-100,1.5 point, p, 2, t, 400

\section{RESULTS AND DISCUSSION}

The advances in solver capabilities have made many Hugoniots easily attainable. All Hugoniots presented in this work required extensive manual manipulation to work in Cheetah 3.0, and/or the Hugoniots required the addition of a substantial amount of Argon to improve numerical stability. Both of these solutions under Cheetah 3.0 were unsatisfactory because either they required an expert user or chemical modification of the system. Experimental data is from LANL shock data [7] and the Russian shock database [8]. Results are presented in Fig. 1-2. Agreement with experiment is quite good (and better than in Cheetah 3.0), although the kink in the $\mathrm{Fe}_{2} \mathrm{O}_{3}$ is slightly too low of a pressure. The success in reproducing known Hugoniots provides confidence in its ability to calculate properties of system without experiments.

\section{CONCLUSIONS}

The Cheetah code has advanced substantially from version 3.0 to version 4.0. We improved the thermodynamic engine and solver system in Cheetah. The improved engine can find a wider range of states, including states with little or no gas. Version 2.0 of the exponential-6 EOS has improved accuracy of the computed results. Many Hugoniots are now easily and accurately calculated in Cheetah 4.0, that were not calculatable in Cheetah 3.0. The success in reproducing known Hugoniots provides us with confidence in Cheetah's ability to calculate properties of systems for which the experimental data is not known. 

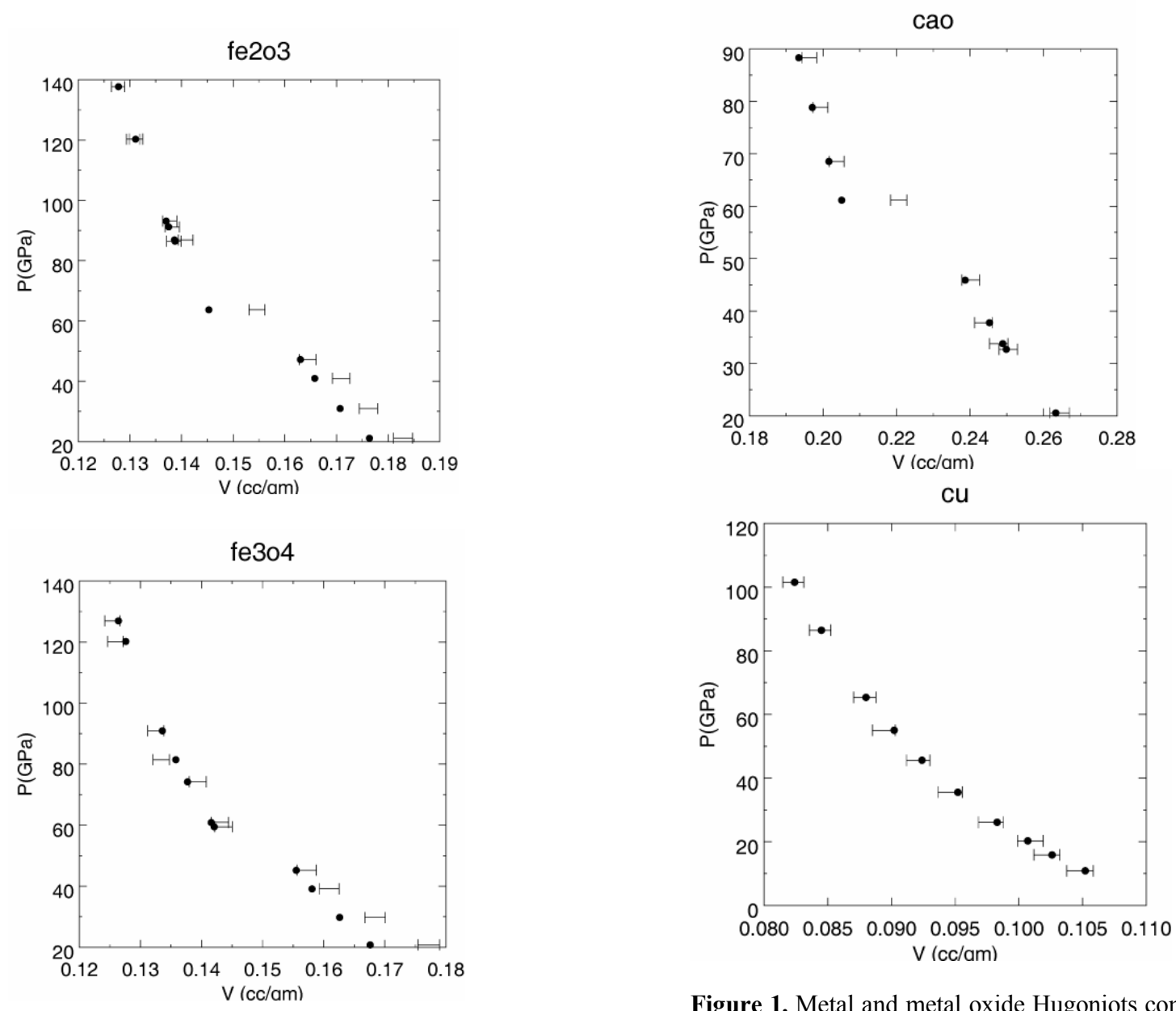

ti

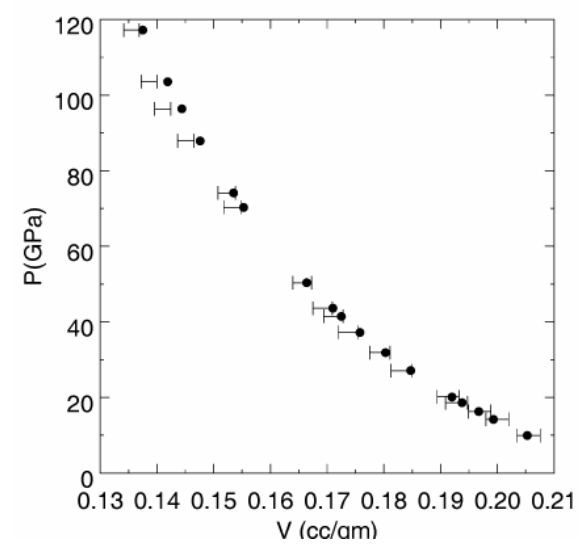

Figure 1. Metal and metal oxide Hugoniots compared to experimental data, calculated with Cheetah 4.0.

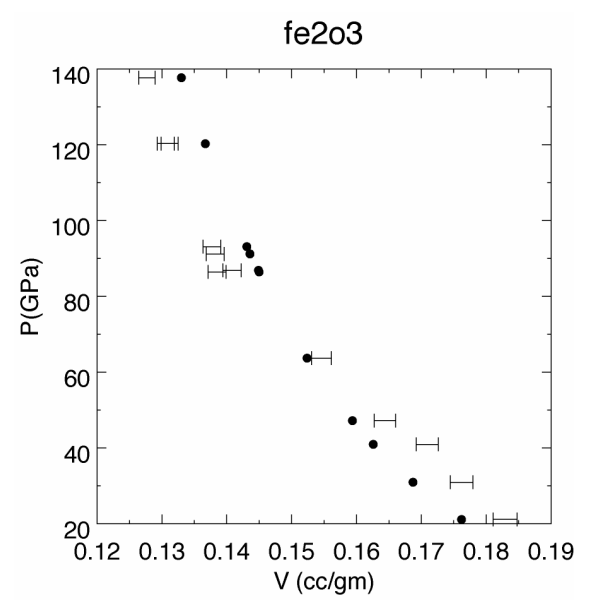




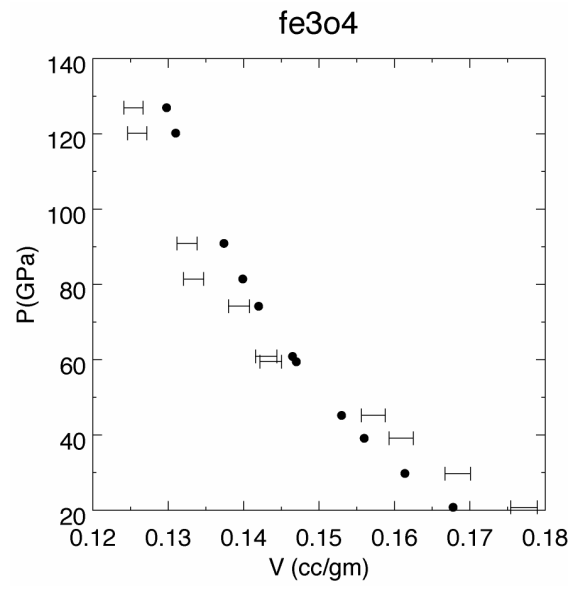

ti

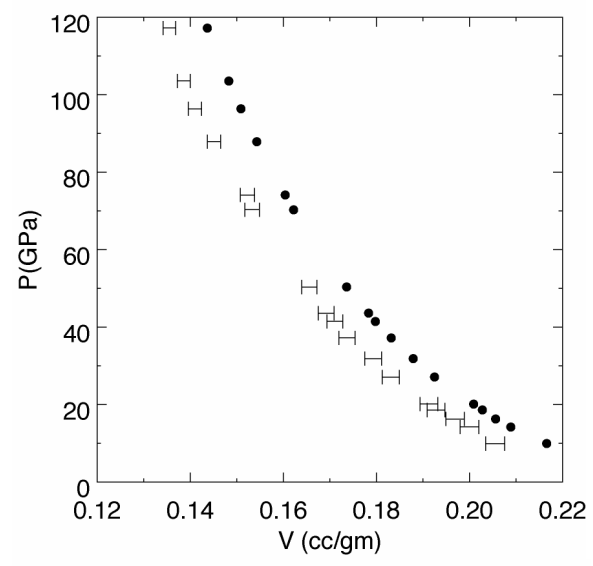

cao

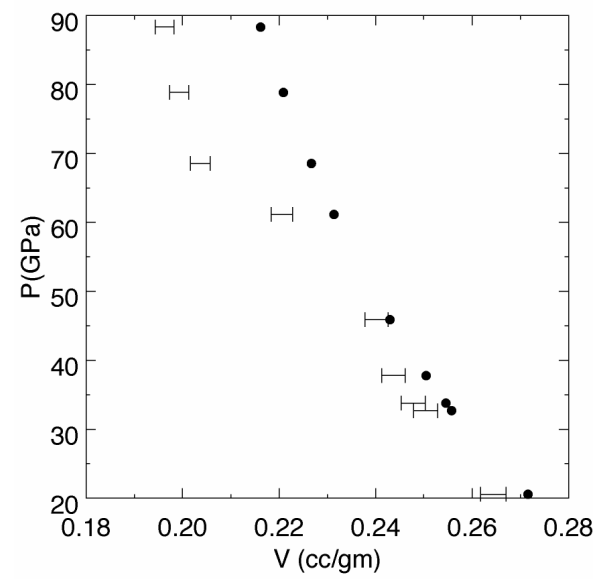

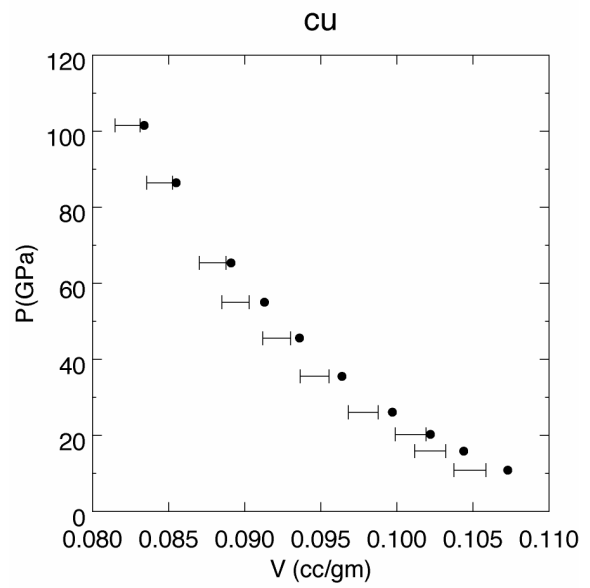

Figure 2. Metal and metal oxide Hugoniots compared to experimental data, calculated with Cheetah 3.0.

\section{ACKNOWLEDGEMENTS}

This work was performed under the auspices of the U.S. Department of Energy by the University of California Lawrence Livermore National Laboratory under contract No. W-7405-Eng-48.

\section{REFERENCES}

1. Fried, L. E., Glaesemann, K. R., Howard W. M Souers. P. C., and Vitello, P. A., UCRL-CODE155944, 2004.

2. Scott H. P., Hemley R. J., Mao, H. K., Herschbach, D, R., Fried, L. E., Howard, W. M., and Bastea, S., Proc. Nat. Acad. Sci. U.S.A. 101, 14023, 2004.

3. Fried, L. E. and Howard, W. M., J Chem. Phys. 109, 7338, 1998.

4. Fried, L.E. and Howard, W. M., Phys. Rev. B 61, 8734, 2000.

5. Spellucci, P., http://plato.la.asu.edu/donlp2.html

6. It is possible to manually obtain this state in 3.0.

7. March, S. P., Ed., LASL Shock Hugoniot Data. University of California Press. 1980.

8. Bushman, A. V., Lomonosov, I. V., Khishchenko, K. V., Kogan, V. E., Levashov, P. R., and Lomov, I. N., http://teos.ficp.ac.ru/rusbank/ 